\title{
Wavelet Density Estimation and Statistical Evidences Role for a GARCH Model in the Weighted Distribution
}

\author{
Mohammad Abbaszadeh, Mahdi Emadi \\ Department of Statistics, Ferdowsi University of Mashhad, Mashhad, Iran \\ Email: abbaszadeh.mo@stu-mail.um.ac.ir,emadi@um.ac.ir
}

Received November 10, 2012; revised December 10, 2012; accepted December 17, 2012

\begin{abstract}
We consider $n$ observations from the GARCH-type model: $Z=U Y$, where $U$ and $Y$ are independent random variables. We aim to estimate density function $Y$ where $Y$ have a weighted distribution. We determine a sharp upper bound of the associated mean integrated square error. We also make use of the measure of expected true evidence, so as to determine when model leads to a crisis and causes data to be lost.
\end{abstract}

Keywords: Density Estimation; GARCH Model; Weighted Distribution; Wavelets; Statistical Evidences; Strongly Mixing

\section{Introduction}

We suppose that $Z_{1}, \cdots, Z_{n}$ is a sample of a strictly stationary and exponentially strongly mixing process $\left(Z_{i}\right)_{i \in \mathbb{Z}}$ where, for any $i \in \mathbb{Z}$,

$$
Z_{i}=U_{i} Y_{i},
$$

$\left(U_{i}\right)_{i \in \mathbb{Z}}$ is a sequence of identically distributed random variables with common known density $f_{U}$ and $\left(Y_{i}\right)_{i \in \mathbb{Z}}$ is a sequence of identically distributed random variables with common unknown density $f_{Y}$. For any $i \in \mathbb{Z}, U_{i}$ and $Y_{i}$ are independent. We suppose that $f_{Y}$ is a weighted density of the form

$$
f_{Y}(x)=\frac{w(x) f_{X}(x)}{\theta}, x \in[0,1],
$$

where $w$ is a known positive function, $f_{X}$ an unknown density of a random variable $X$ and $\theta$ is the unknown normalization parameter:

$$
\theta=\mathbb{E}(w(X))=\int_{0}^{1} w(x) f_{X}(x) \mathrm{d} x .
$$

Our goal is to estimate $f_{X}$ when only $Z_{1}, \cdots, Z_{n}$ are observed. The Equation (1) is a GARCH-type time series model classically encountered in financial models see [1] and practical examples of Equation (2) can be found in e.g. [2-4].

In this article, we construct a linear wavelet estimator and measure its performance by determining upper bounds of the mean integrated squared error (MISE) over Besov space.

In what follows, we have also surveyed the role of data and evidential inference in the model. The data play a very important essential role in statistical analysis, to the extent that many statistical researchers believe in the famous saying: "Ask the data." We consider the Test

$$
\left\{\begin{array}{l}
H_{1}: \theta=\theta_{1} \\
H_{2}: \theta=\theta_{2}=\alpha \theta_{1}(\alpha>1),
\end{array}\right.
$$

for the model and we evaluate the sensitivity of the value in the test hypotheses. In this test, the evaluation criterion is the area between the curves of the cumulative distribution functions under $H_{1}$ and $H_{2}$ hypotheses. Details on evidential inference can be found in [5,6]. Also [7] have studied about Comparing of record data and random observation based on statistical evidence.

Through the rest of the paper, at first assumptions and then an introduction about wavelets are presented in Section 2. The estimators and results are given in Section 3. In Section 4, general explanations regarding evidential inference and its application in a test. The proofs are gathered in Section 5.

\section{Assumptions and Wavelets}

\subsection{Assumptions}

We formulate the following assumptions:

- Without loss of generality, we assume that $f_{X}$ and $f_{Y}$ have the support $[0,1]$ and $f_{X} \in \mathbb{L}^{2}([0,1])$ where

$$
\mathbb{L}^{2}([0,1])=\left\{g:[0,1] \rightarrow \mathbb{R} ;\left(\int_{0}^{1} g^{2}(x) \mathrm{d} x\right)^{1 / 2}<\infty\right\} .
$$


- We suppose that for any $m \in \mathbb{Z}$, the $m$-th strongly mixing coefficient of $\left(Z_{i}\right)_{i \in \mathbb{Z}}$ by

$$
a_{m}=\sup _{(A, B) \in F_{-\infty, 0}^{Z} \times F_{m, \infty}^{Z}}|\mathbb{P}(A \cap B)-\mathbb{P}(A) \mathbb{P}(B)|,
$$

where, for any $u \in \mathbb{Z}$, let $F_{-\infty, u}^{Z}$ be the $\sigma$-algebra generated by $\cdots, Z_{u-1}, Z_{u}$ and $F_{u, \infty}^{Z}$ is the $\sigma$-algebra generated by $Z_{u}, Z_{u+}, \cdots$.

We suppose that there exist three (known) constants, $\gamma>0, c>0$ and $\theta>0$ such that

$$
a_{m} \leq \gamma \exp \left(-c|m|^{\theta}\right) \text {. }
$$

This assumption is satisfied by a large class of GARCH processes. See e.g. [8-10].

- For any $x \in[0,1]$, it follows from the independence of $U_{1}$ and $Y_{1}$ that the density of $Z_{1}$ is

$$
f_{Z}(x)=\int_{x}^{1} f_{U}\left(\frac{x}{y}\right) f_{Y}(y) \frac{1}{y} \mathrm{~d} y .
$$

- We suppose that there exists two constants, $C>0$ and $c>0$, such that

$$
\sup _{x \in[0,1]} f_{Z}(x) \leq C .
$$

and

$$
\sup _{x \in[0,1]} w(x) \leq C, \sup _{x \in[0,1]} w^{\prime}(x) \leq C, \inf _{x \in[0,1]} w(x) \geq c .
$$

\subsection{Wavelets and Besov Balls}

Let $N$ be a positive integer, and $\phi$ and $\psi$ be the Daubechies wavelets $d b N$ which satisfy

$$
\operatorname{supp}(\phi)=\operatorname{supp}(\psi)=1-N, N \text {. }
$$

Set

$$
\begin{aligned}
& \phi_{j, k}(x)=2^{j / 2} \phi\left(2^{j} x-k\right), \\
& \psi_{j, k}(x)=2^{j / 2} \psi\left(2^{j} x-k\right) .
\end{aligned}
$$

Then, there exists an integer $\tau$ such that, for any integer $\ell \geq \tau$, the collection

$$
\begin{aligned}
\mathcal{B}= & \left\{\phi_{\ell, k}(\cdot), k \in\left\{0, \cdots, 2^{\ell}-1\right\} ; \psi_{j, k}(\cdot) ;\right. \\
& \left.j \in \mathbb{N}-\{0, \cdots, \ell-1\}, k \in\left\{0, \cdots, 2^{j}-1\right\}\right\}
\end{aligned}
$$

is an orthonormal basis of $\mathbb{L}^{2}[0,1]$ (the space of squareintegrable functions on 0,1$)$. We refer to [11].

For any integer $\ell \geq \tau$, any $h \in \mathbb{L}^{2}[0,1]$ can be expanded on $\mathcal{B}$ as

$$
h(x)=\sum_{k=0}^{2^{\ell}-1} \alpha_{\ell, k} \phi_{\ell, k}(x)+\sum_{j=\ell}^{\infty} \sum_{k=0}^{2^{j}-1} \beta_{j, k} \psi_{j, k}(x), x \in[0,1],
$$

where $\alpha_{j, k}$ and $\beta_{j, k}$ are the wavelet coefficients of $h$ defined by

$$
\begin{aligned}
\alpha_{j, k} & =\int_{0}^{1} h(x) \phi_{j, k}(x) \mathrm{d} x, \beta_{j, k} \\
& =\int_{0}^{1} h(x) \psi_{j, k}(x) \mathrm{d} x .
\end{aligned}
$$

Let $M>0, s>0, p \geq 1$ and $r \geq 1$. A function $h$ belongs to $B_{p, r}^{s}(M)$ if and only if there exists a constant $M^{*}>0$ (depending on $M$ ) such that the associated wavelet coefficients Equation (6) satisfy

$$
\left(\sum_{j=\tau-1}^{\infty}\left(2^{j(s+1 / 2-1 / p)}\left(\sum_{k=0}^{2^{j}-1}\left|\beta_{j, k}\right|^{p}\right)^{1 / p}\right)^{r}\right)^{1 / r} \leq M^{*} .
$$

We set $\beta_{\tau-1, k}=\alpha_{\tau, k}$. Details on Besov balls can be found in [12].

\section{Estimators and Results}

Firstly, we consider the following estimator for $\theta$

$$
\hat{\theta}=\left(\frac{1}{n} \sum_{i=1}^{n} \frac{w\left(Z_{i}\right)-Z_{i} w^{\prime}\left(Z_{i}\right)}{w^{2}\left(Z_{i}\right)}\right)^{-1} .
$$

Then, for any integer $j \geq \tau$ and any $k \in\left\{0, \cdots, 2^{j}-1\right\}$, we estimate

$$
\alpha_{j, k}=\int_{0}^{1} f_{X}(x) \phi_{j, k}(x) \mathrm{d} x, \hat{\alpha}_{j, k}=\frac{\hat{\theta}}{n} \sum_{i=1}^{n} T\left(\phi_{j, k}\right)\left(Z_{i}\right),
$$

where, for any $h: \mathcal{C}[0,1] \rightarrow \mathbb{R}, T$ is the operator

$$
T(h)(x)=\frac{h(x) w(x)+x h^{\prime}(x) w(x)-x h(x) w^{\prime}(x)}{w^{2}(x)} .
$$

$\hat{\alpha}_{j, k}$ and $\hat{\theta}$ are similar with multiplicative censoring model (see [13]).

We are now in the position to define the considered estimators for $f_{X}$. Suppose that $f_{X} \in B_{p, r}^{s}(H)$. We define the linear estimator $\hat{f}$ by

$$
\hat{f}(x)=\sum_{k=0}^{2^{j} 0-1} \hat{\alpha}_{j_{0}, k} \phi_{j_{0}, k}(x), x \in[0,1],
$$

where $\hat{\alpha}_{j, k}$ is defined by Equation (8) and $j_{0}$ is the integer satisfying

$$
(1 / 2) n^{1 /(2 s+q+3)}<2^{j_{0}} \leq n^{1 /(2 s+q+3)} .
$$

\section{Lemma 3.1}

- Let $\hat{\theta}$ be Equation (7) and $\theta=\int_{0}^{1} w(x) f_{X}(x) \mathrm{d} x$. Then we have

$$
\mathbb{E}\left(\frac{1}{\hat{\theta}}\right)=\frac{1}{\theta} .
$$

- Let $Z_{1}, \cdots, Z_{n}$ be Equation (1), $T$ be Equation (9) 
and for any integer $j \geq \tau$ and any $k \in\left\{0, \cdots, 2^{j}-1\right\}$, $\alpha_{j, k}=\int_{0}^{1} f_{X}(x) \phi_{j, k}(x) \mathrm{d} x$. Then we have

$$
\mathbb{E}\left(\frac{\theta}{n} \sum_{i=1}^{n} T\left(\phi_{j, k}\right)\left(Z_{i}\right)\right)=\alpha_{j, k} .
$$

- For any integer $j \geq \tau$ and any $k \in\left\{0, \cdots, 2^{j}-1\right\}$, let $\hat{\alpha}_{j, k}$ be Equation (8) and $\alpha_{j, k}=\int_{0}^{1} f_{X}(x) \phi_{j, k}(x) \mathrm{d} x$.

Then, under the assumptions of Subsection 2.1, there exists a constant $C>0$ such that

$$
\left|\hat{\alpha}_{j, k}-\alpha_{j, k}\right| \leq C\left(\left|\frac{\theta}{n} \sum_{i=1}^{n} T\left(\phi_{j, k}\right)\left(Z_{i}\right)-\alpha_{j, k}\right|+\left|\frac{1}{\hat{\theta}}-\frac{1}{\theta}\right|\right) \text {. }
$$

Proposition 3.1 For any integer $j \geq \tau$ and any $k \in\left\{0, \cdots, 2^{j}-1\right\}$ Then, under the assumptions of Subsection 2.1 ,

- $\sup _{x \in[0,1]}\left|T\left(\phi_{j_{0}, k}\right)(x)\right| \leq C 2^{\frac{3}{2} j}$.

- $\mathbb{E}\left(T\left(\phi_{j_{0}, k}\right)\left(Z_{0}\right)\right)^{2} \leq C 2^{2 j}$.

Proposition 3.2 Let $q \in(0,1)$, for any integer $j \geq \tau$ and any $k \in\left\{0, \cdots, 2^{j}-1\right\}$, let $\hat{\alpha}_{j, k}$ be Equation (8) and $\alpha_{j, k}=\int_{0}^{1} f_{X}(x) \phi_{j, k}(x) \mathrm{d} x$. Then,

- there exists a constant $C>0$ such that

$$
\mathbb{V}\left(\frac{\theta}{n} \sum_{i=1}^{n} T\left(\phi_{j, k}\right)\left(Z_{i}\right)\right) \leq \frac{C}{n} 2^{j(2+q)} .
$$

- there exists a constant $C>0$ such that

$$
\mathbb{V}\left(\frac{1}{\hat{\theta}}\right) \leq \frac{C}{n}
$$

- there exists a constant $C>0$ such that

$$
\mathbb{E}\left(\left(\hat{\alpha}_{j, k}-\alpha_{j, k}\right)^{2}\right) \leq \frac{C}{n} 2^{j(2+q)} .
$$

Theorem 3.1 (Upper bound for $\hat{\boldsymbol{f}}$ ) Consider Equation (1) under the assumptions of Subsection 2.1. Suppose that $f_{X} \in B_{p, r}^{s}(H)$ with $s>0, p \geq 2, r \geq 1$. For any $q \in(0,1)$ and $\hat{f}$ be Equation (10), then there exists a constant $C>0$ such that

$$
\mathbb{E}\left(\int_{0}^{1}\left(\hat{f}(x)-f_{X}(x)\right)^{2} \mathrm{~d} x\right) \leq C n^{-2 s /(2 s+q+3)} .
$$

Remark that $n^{-2 s /(2 s+q+3)}$ is the slower than the optimal one in the standard density estimation problem i.e. $n^{-2 s /(2 s+1)}$ (see e.g. [14, Chapter 10]). This deterioration is due to the presence of GARCH model and weighted distribution.

\section{Statistical Evidence}

\subsection{Statistical Inference}

The evidential approach to statistical inference concerns a novel approach in statistical analysis. Evidential inference is solely based on data as evidence and calculation of the evidence strength. It is not influenced by mental and personal components and factors such as former beliefs and loss functions. Using evidential inference in the model Equation (1), we will survey when censoring of data will lead to considerable data loss, and we will determine the time when data is lost by determining an appropriate criterion. In the model $Z_{i}=U_{i} Y_{i}$ for $i \in\{1, \cdots, n\}$, the data observed from the variable $Z_{i}$ are denoted by the subscript (cen), and the data observed from the variable $Y_{i}$ are denoted by the subscript (ncen). Considering the Test Equation (3) in the above model, due to the symmetry of the test hypotheses in evidential methods and without losing the generality of the problem, the value of is assumed to be $\alpha>1$. In order to support $H_{1}$ and $H_{2}$ hypotheses, we now use the following criterion:

$$
\boldsymbol{\gamma}=\frac{\boldsymbol{a} \boldsymbol{b} \boldsymbol{c}_{c e n}(\eta)}{\boldsymbol{a} \boldsymbol{b} \boldsymbol{c}_{\text {ncen }}(\eta)}
$$

where $\boldsymbol{a} \boldsymbol{b} \boldsymbol{c}_{c e n}(\eta)$ and $\boldsymbol{a} \boldsymbol{b} \boldsymbol{c}_{n c e n}(\eta)$ are the measure of expected true evidence in the censored and uncensored data respectively, and $\eta$ is the criterion of the support of data from $H_{1}$ hypothesis against $H_{2}$ hypothesis. This support criterion is optimal when the area between the two curves of $\eta$ cumulative functions under $H_{1}$ and $H_{2}$ is maximum, please see [15]. This area which is denoted by $\boldsymbol{a b c}(\eta)$ in the form of

$$
\begin{aligned}
\boldsymbol{a b c}(\eta) & =\int_{0}^{1} \boldsymbol{\kappa}_{2}(\eta) \mathrm{d} \eta-\int_{0}^{1} \kappa_{1}(\eta) \mathrm{d} \eta \\
& =\int_{0}^{1}\left(1-\kappa_{1}(\eta)\right) \mathrm{d} \eta-\int_{0}^{1}\left(1-\kappa_{2}(\eta)\right) \mathrm{d} \eta \\
& =\boldsymbol{E}_{1}(\eta)-\boldsymbol{E}_{2}(\eta),
\end{aligned}
$$

where $\kappa_{i}(\eta)$ is the cumulative distribution function of $\eta$ and $\boldsymbol{E}_{i}(\eta)$ is the mean value of $\eta$ under $H_{i}:(i=1,2)$ hypotheses. In view of [6], the support criterion $\eta$ is defined as follows:

$$
\eta=\frac{\lambda}{1+\lambda},
$$

where $\lambda$ is the likelihood ratio and for the two censored and uncensored cases we have

$$
\lambda_{c e n}=\frac{f_{Z}^{\theta 1}(\underline{z})}{f_{Z}^{\theta 2}(\underline{z})} \text { and } \lambda_{\text {ncen }}=\frac{f_{Y}^{\theta 1}(\underline{y})}{f_{Y}^{\theta 2}(\underline{y})},
$$

where $f_{Z}^{\theta i}(\underline{z})$ and $f_{Y}^{\theta i}(\underline{y})$ are likelihood functions 
for $Z_{i}$ and $Y_{i}$ variables respectively, in the Equation (1) under $H_{i}:(i=1,2)$ hypotheses.

\subsection{Measuring Statistical Evidence}

We consider i.i.d case for variables in the Equations (1) and (2), also set $w(x)=1$ and then, we investigate the behavior of $\alpha$ by means of simulation. In addition, we analyze $H_{1}$ and $H_{2}$ hypotheses in Test Equation (3) by determining support criterion $\eta$ of the measure of expected true evidence $\boldsymbol{a b c}(\eta)$. The programming codes of this part are written in the MAPLE (15) environment.

Example 1. In this example, we generate data from gamma and uniform distribution as follows, considering multiplicative censoring model:

$$
\begin{aligned}
& Y_{i} \sim \operatorname{Gamma}\left(2, \theta_{i}\right) \text { and } U_{i} \sim \operatorname{Uniform}(0,1) \\
& \text { and } H_{i}: \theta=\theta_{i} \text { for }(i=1,2)
\end{aligned}
$$

Then, according to Equations (13) and (14), we calculate the support criterion and the likelihood ratio via below relations:

$$
\eta_{c e n}=\frac{\lambda_{c e n}}{1+\lambda_{c e n}}
$$

such that

$$
\lambda_{\text {cen }}=\frac{f_{Z}^{\theta_{1}}(\underline{z})}{f_{Z}^{\theta_{2}}(\underline{z})}=\left(\frac{\theta_{2}}{\theta_{1}}\right)^{n} \mathrm{e}^{-\sum_{i=1}^{n} z_{i}\left(\frac{1}{\theta_{1}}-\frac{1}{\theta_{2}}\right)} .
$$

and

such that

$$
\eta_{\text {ncen }}=\frac{\lambda_{\text {ncen }}}{1+\lambda_{\text {ncen }}}
$$

$$
\lambda_{\text {ncen }}=\frac{f_{Y}^{\theta_{1}}(\underline{y})}{f_{Y}^{\theta_{2}}(\underline{y})}=\left(\frac{\theta_{2}}{\theta_{1}}\right)^{2 n} \mathrm{e}^{-\sum_{i=1}^{n} y_{i}\left(\frac{1}{\theta_{1}}-\frac{1}{\theta_{2}}\right)} .
$$

For different values of $\alpha$ and $n$, we calculate the value of $\gamma$ according to Equation (12). The results can be observed in Table 1. By carefully considering this table, it is observed that as the value of $\alpha$ increases (which implies the distance growth between $\theta_{1}$ and $\theta_{2}$ ), the value of $\gamma$ gets closer to one. In other words, $\boldsymbol{a} \boldsymbol{b} \boldsymbol{c}_{\text {cen }}$ approaches $\boldsymbol{a} \boldsymbol{b} \boldsymbol{c}_{\text {ncen }}$ more and more. This fact can be interpreted in this way that if the distance between $\theta_{1}$ and $\theta_{2}$ is large, the data lost in censored data $Y_{i}$ is negligible. That is to say, evidential inference draws our attention to the time when $\theta_{2}$ is close to $\theta_{1}$. The above analysis can also be observed in Figure 1.

In what follows, the variations of sample volume ratio increase against $\alpha$ are investigated, and the value of $\alpha$ in the $\operatorname{ArgMax} \frac{\gamma\left(n_{2}, \alpha\right)}{\gamma\left(n_{1}, \alpha\right)}$ equation is determined for different values of $n_{1}$ and $n_{2}$.

$$
\text { If } \frac{n_{2}}{n_{1}}=\frac{25}{20}=1.25 \text { then } \alpha=1.6 \text {. The result can be }
$$

viewed in Figure 2, If $\frac{n_{2}}{n_{1}}=\frac{15}{10}=1.5$ then $\alpha=2.1$.

The result can be viewed in Figure 3, If $\frac{n_{2}}{n_{1}}=\frac{20}{10}=2$

then $\alpha=2.5$. The result can be viewed in Figure 4 .

The above results can be interpreted in this way that as the sample volume increases from a certain stage on, the value of $\alpha$ remains constant. In other words, it can be intuitively said that the $\operatorname{ArgMax} \frac{\gamma\left(n_{2}, \alpha\right)}{\gamma\left(n_{1}, \alpha\right)}$ ratio tends to the constant $\alpha$ value, which this also leads to an increase in evidential strength.

\section{Proofs}

In this section, $C$ denotes any constant that does not depend on $j, k$ and $n$. Its value may change from one term to another and may depends on $\phi$ or $\psi$.

Table 1. Computed values for $\gamma$.

\begin{tabular}{ccccccccc}
\hline n value & \multicolumn{7}{c}{$\alpha$ value } \\
\hline \multicolumn{10}{c}{} & $\alpha=1.1$ & $\alpha=1.6$ & $\alpha=2.1$ & $\alpha=2.6$ & $\alpha=3.1$ & $\alpha=3.6$ & $\alpha=4.1$ \\
10 & 0.5122 & 0.6427 & 0.7580 & 0.8398 & 0.8994 & 0.9317 & 0.9596 \\
15 & 0.5165 & 0.6879 & 0.8273 & 0.9141 & 0.9572 & 0.9799 & 0.9894 \\
20 & 0.5291 & 0.7251 & 0.8773 & 0.9516 & 0.9824 & 0.9931 & 0.9970 \\
25 & 0.5219 & 0.7614 & 0.9145 & 0.9738 & 0.9923 & 0.9981 & 0.9991 \\
30 & 0.5329 & 0.7881 & 0.9409 & 0.9862 & 0.9968 & 0.9992 & 0.9995 \\
\hline
\end{tabular}

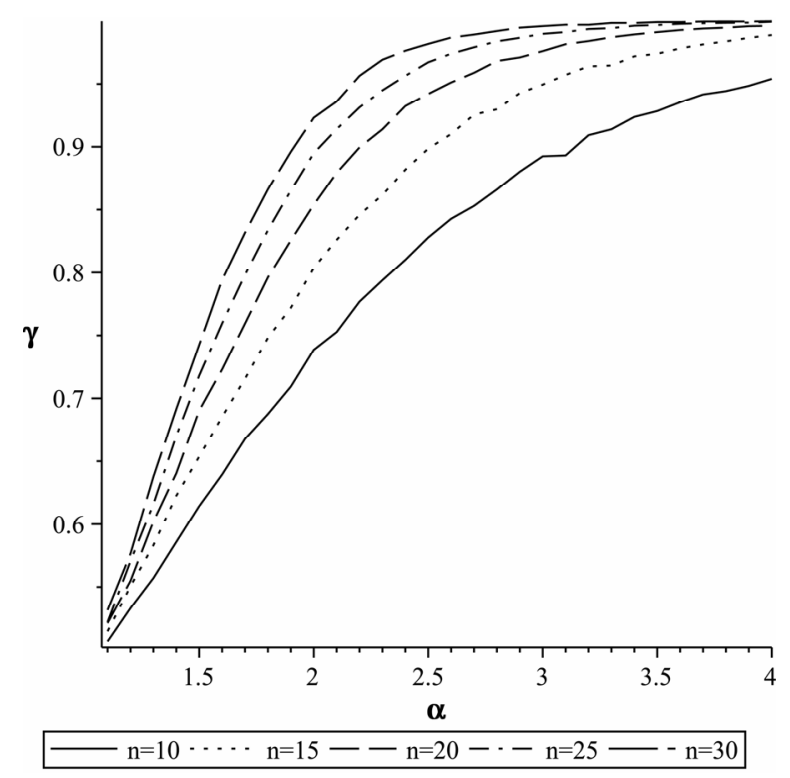

Figure 1. $\gamma$ computed from gamma distribution for different values of $n$. 


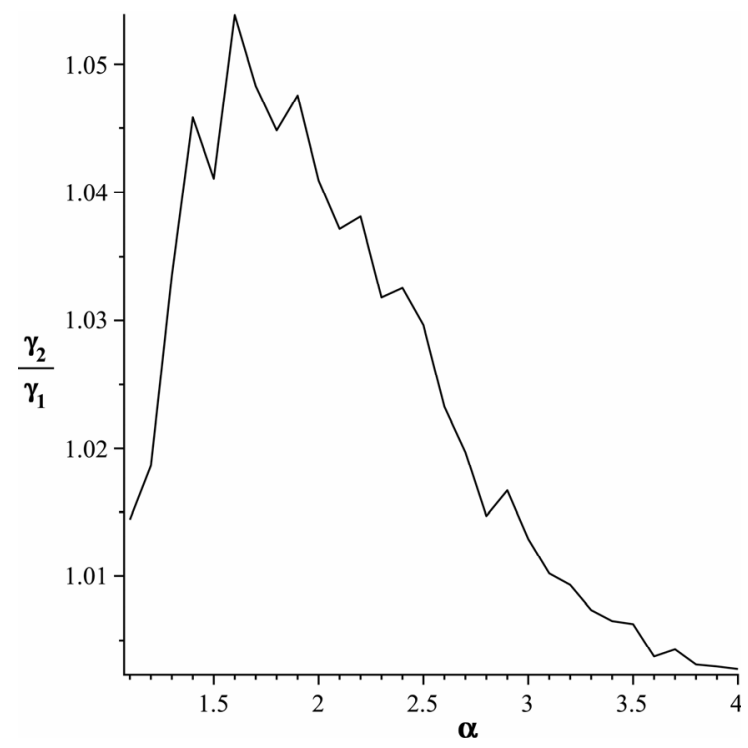

Figure 2. The most relative changes when sample size $n_{1}=$ 20 increases to $n_{2}=25$ happens in $\alpha=1.6$.

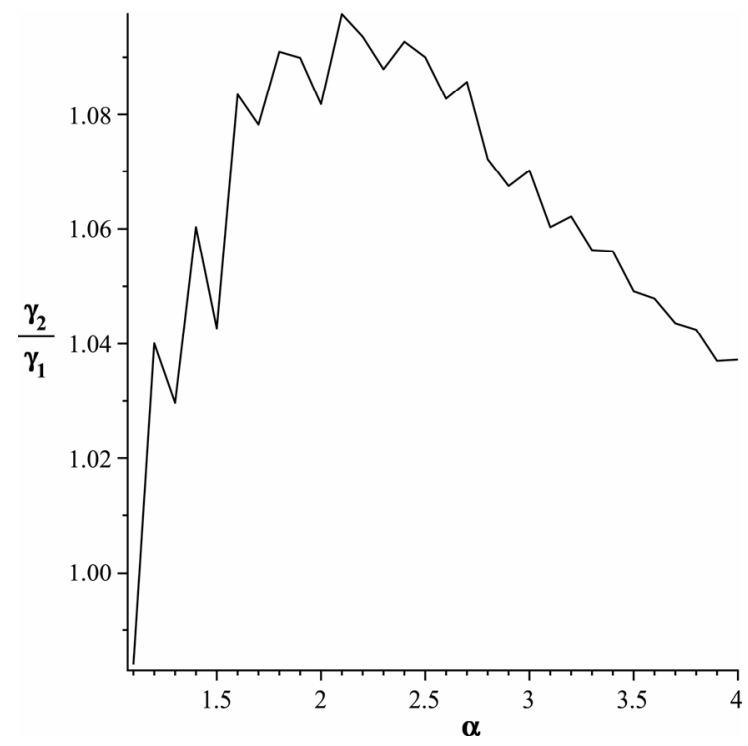

Figure 3. The most relative changes when sample size $n_{1}=$ 10 increases to $n_{2}=15$ happens in $\alpha=2.1$.

Proof of Lemma 3.1.

Proof can be found in [13].

Proof of proposition 3.1.

1. By Equation (9) and Equation (5), we have

$$
\begin{aligned}
& \left|T\left(\phi_{j, k}\right)(x)\right| \\
& \leq \frac{1}{w^{2}(x)}\left(\begin{array}{l}
\left.\left|\phi_{j, k}(x) w(x)\right|+\left|x\left(\phi_{j, k}\right)^{\prime}(x) w(x)\right|\right) \\
+\left|x \phi_{j, k}(x) w^{\prime}(x)\right|
\end{array}\right) \\
& \leq C\left(\left|\phi_{j, k}(x)\right|+\left|\left(\phi_{j, k}\right)^{\prime}(x)\right|\right) .
\end{aligned}
$$

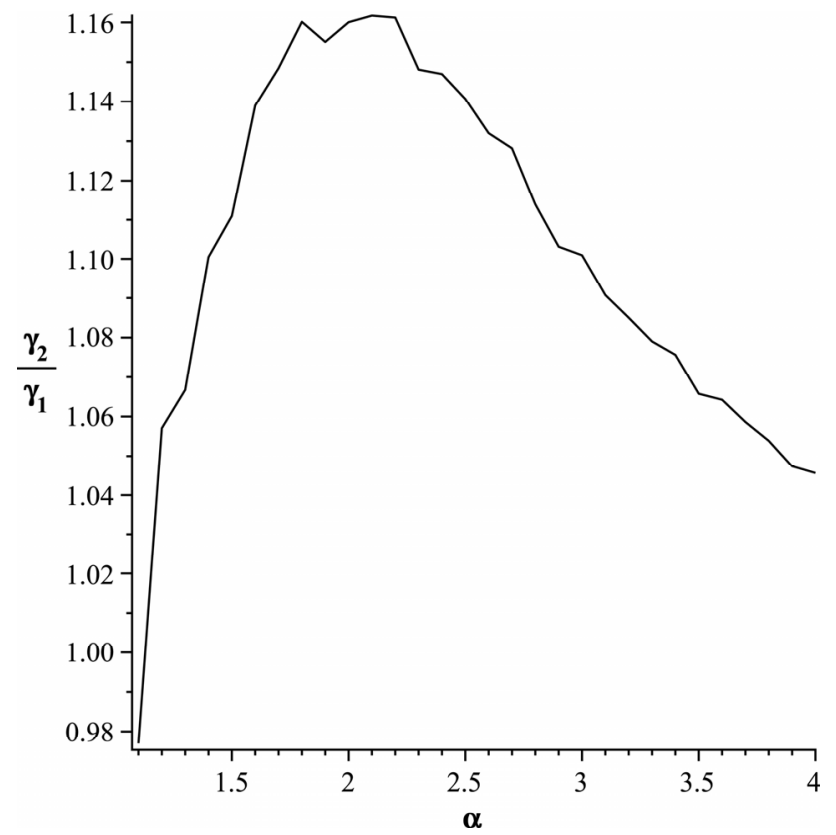

Figure 4. The most relative changes when sample size $n_{1}=$ 10 increases to $n_{2}=20$ happens in $\alpha=2.2$.

Therefore

$$
\begin{aligned}
& \sup _{x \in[0,1]}\left|T\left(\phi_{j_{0}, k}\right)(x)\right| \\
& \leq C\left(\sup _{x \in[0,1]}\left(\phi_{j_{0}, k}\right)(x)+\sup _{x \in[0,1]}\left(\phi_{j_{0}, k}\right)^{\prime}(x)\right) \\
& \leq C\left(2^{\frac{j}{2}}+2^{\frac{3}{2} j}\right) \leq C 2^{\frac{3}{2} j} .
\end{aligned}
$$

2. By Equation (9) and Equation (5), we have

$$
\begin{aligned}
& \mathbb{E}\left(\left|T\left(\phi_{j, k}\right)\left(Z_{0}\right)\right|^{2}\right) \\
& \leq C\left(\mathbb{E}\left(\left|\phi_{j, k}\left(Z_{0}\right)\right|^{2}\right)+\mathbb{E}\left(\left|\left(\phi_{j, k}\right)^{\prime}\left(Z_{0}\right)\right|^{2}\right)\right) .
\end{aligned}
$$

By Equation (4) and under the assumptions of Subsection 2.1 and also doing the change of variables $y=2^{j} x-k$, we have

$$
\begin{aligned}
& \mathbb{E}\left(\left|\phi_{j, k}\left(Z_{0}\right)\right|^{2}\right) \\
& =\int_{0}^{1}\left|\phi_{j, k}(x)\right|^{2} f_{Z}(x) \mathrm{d} x \leq C \int_{0}^{1}\left|\phi_{j, k}(x)\right|^{2} \mathrm{~d} x \\
& =C \int_{-k}^{2^{j}-k}|\phi(y)|^{2} \mathrm{~d} y \leq C .
\end{aligned}
$$

Using again Equation (4) and the assumptions of Subsection 2.1, the equality $\left(\phi_{j, k}\right)^{\prime}(x)=2^{3 j / 2} \phi^{\prime}\left(2^{j} x-k\right)$ and doing the change of variables $y=2^{j} x-k$, we ob- 
tain

$$
\begin{aligned}
& \mathbb{E}\left(\left|\left(\phi_{j, k}\right)^{\prime}\left(Z_{0}\right)\right|^{2}\right) \\
& =\int_{0}^{1}\left|\left(\phi_{j, k}\right)^{\prime}(x)\right|^{2} f_{Z}(x) \mathrm{d} x \leq C \int_{0}^{1}\left|\left(\phi_{j, k}\right)(x)\right|^{2} \mathrm{~d} x \\
& \leq C 2^{2 j} \int_{-k}^{2^{j}-k}\left|\phi^{\prime}(y)\right|^{2} \mathrm{~d} y \leq C 2^{2 j} .
\end{aligned}
$$

Combining Equations (17)-(19), we obtain

$$
\mathbb{E}\left(\left|T\left(\phi_{j, k}\right)\left(Z_{0}\right)\right|^{2}\right) \leq C 2^{2 j}
$$

The proof of Proposition 3.1 is complete.

\section{Proof of Proposition 3.2.}

1. We have

$$
\begin{aligned}
& \mathbb{V}\left(\frac{\theta}{n} \sum_{i=1}^{n} T\left(\phi_{j, k}\right)\left(Z_{i}\right)\right) \\
& =\frac{\theta^{2}}{n^{2}} \sum_{v=1}^{n} \sum_{\ell=1}^{n} \mathbb{C}\left(T\left(\phi_{j_{0}, k}\right)\left(Z_{v}\right), T\left(\phi_{j_{0}, k}\right)\left(Z_{\ell}\right)\right) \\
& =\frac{\theta^{2}}{n^{2}} \mathbb{V}\left(T\left(\phi_{j, k}\right)\left(Z_{1}\right)\right) \\
& +2 \frac{\theta^{2}}{n^{2}} \sum_{v=2}^{n} \sum_{\ell=1}^{v-1} \mathbb{C}\left(T\left(\phi_{j_{0}, k}\right)\left(Z_{v}\right), T\left(\phi_{j_{0}, k}\right)\left(Z_{\ell}\right)\right) \\
& \leq \frac{\theta^{2}}{n^{2}} \mathbb{V}\left(T\left(\phi_{j, k}\right)\left(Z_{1}\right)\right) \\
& +2 \frac{\theta^{2}}{n^{2}} \mid \sum_{v=2}^{n} \sum_{\ell=1}^{v-1} \mathbb{C}\left(T\left(\phi_{j_{0}, k}\right)\left(Z_{v}\right), T\left(\phi_{j_{0}, k}\right)\left(Z_{\ell}\right)\right) .
\end{aligned}
$$

Using Equation (20) we obtain

$$
\mathbb{V}\left(T\left(\phi_{j, k}\right)\left(Z_{1}\right)\right) \leq \mathbb{E}\left(\left(T\left(\phi_{j, k}\right)\left(Z_{1}\right)\right)^{2}\right) \leq C 2^{2 j} .
$$

also

$$
\begin{aligned}
& \left|\sum_{v=2}^{n} \sum_{\ell=1}^{v-1} \mathbb{C}\left(T\left(\phi_{j_{0}, k}\right)\left(Z_{v}\right), T\left(\phi_{j_{0}, k}\right)\left(Z_{\ell}\right)\right)\right| \\
& =\left|\sum_{m=1}^{n}(n-m) \mathbb{C}\left(T\left(\phi_{j_{0}, k}\right)\left(Z_{0}\right), T\left(\phi_{j_{0}, k}\right)\left(Z_{m}\right)\right)\right| \\
& \leq n \sum_{m=1}^{n}\left|\mathbb{C}\left(T\left(\phi_{j_{0}, k}\right)\left(Z_{0}\right), T\left(\phi_{j_{0}, k}\right)\left(Z_{m}\right)\right)\right|
\end{aligned}
$$

By the Davydov inequality (see [16]) and for any $q \in(0,1)$ we obtain

$\mathbb{C}\left(T\left(\phi_{j_{0}, k}\right)\left(Z_{0}\right), T\left(\phi_{j_{0}, k}\right)\left(Z_{m}\right)\right)$

$\leq 10 a_{m}^{q}\left(\sup _{x \in[0,1]}\left|T\left(\phi_{j_{0}, k}\right)(x)\right|\right)^{2 q}\left(\mathbb{E}\left(T\left(\phi_{j_{0}, k}\right)\left(Z_{0}\right)\right)^{2}\right)^{1-q}$.

Putting Equation (24), Equation (16) and Equation (20) together, we have

$$
\begin{aligned}
& \mathbb{C}\left(T\left(\phi_{j_{0}, k}\right)\left(Z_{0}\right), T\left(\phi_{j_{0}, k}\right)\left(Z_{m}\right)\right) \\
& \leq 10 a_{m}^{q} C 2^{3 j q} 2^{2 j(1-q)}=C a_{m}^{q} 2^{j(2+q)} .
\end{aligned}
$$

Since $\sum_{m=1}^{n} a_{m}^{q} \leq \sum_{m=1}^{\infty} a_{m}^{q}=C$, we obtain

$$
\begin{aligned}
& \left|\sum_{v=2}^{n} \sum_{\ell=1}^{v-1} \mathbb{C}\left(T\left(\phi_{j_{0}, k}\right)\left(Z_{v}\right), T\left(\phi_{j_{0}, k}\right)\left(Z_{\ell}\right)\right)\right| \\
& \leq n C 2^{j(2+q)} \sum_{m=1}^{n} a_{m}^{q} \leq n C 2^{j(2+q)} .
\end{aligned}
$$

Putting Equations (21) and (22) and Equation (26) together, we have

$$
\mathbb{V}\left(\frac{\theta}{n} \sum_{i=1}^{n} T\left(\phi_{j, k}\right)\left(Z_{i}\right)\right) \leq \frac{C}{n} 2^{j(2+q)} .
$$

2. We have

$$
\begin{aligned}
& \mathbb{V}\left(\frac{1}{\hat{\theta}}\right)=\mathbb{V}\left(\frac{1}{n} \sum_{i=1}^{n} \frac{w\left(Z_{i}\right)-Z_{i} w^{\prime}\left(Z_{i}\right)}{w^{2}\left(Z_{i}\right)}\right) \\
& =\frac{1}{n} \mathbb{V}\left(\frac{w\left(Z_{i}\right)-Z_{i} w^{\prime}\left(Z_{i}\right)}{w^{2}\left(Z_{i}\right)}\right) \\
& +\frac{2}{n^{2}} \sum_{v=2}^{n} \sum_{\ell=1}^{v-1} \mathbb{C}\left(\frac{w\left(Z_{v}\right)-Z_{v} w^{\prime}\left(Z_{v}\right)}{w^{2}\left(Z_{v}\right)}, \frac{w\left(Z_{\ell}\right)-Z_{\ell} w^{\prime}\left(Z_{\ell}\right)}{w^{2}\left(Z_{\ell}\right)}\right) .
\end{aligned}
$$

By Equation (5), we have

$$
\mathbb{V}\left(\left|\frac{w\left(Z_{i}\right)-Z_{i} w^{\prime}\left(Z_{i}\right)}{w^{2}\left(Z_{i}\right)}\right|\right) \leq C .
$$

By the Davydov inequality (see [16]) we obtain

$$
\mathbb{C}\left(\frac{w\left(Z_{v}\right)-Z_{v} w^{\prime}\left(Z_{v}\right)}{w^{2}\left(Z_{v}\right)}, \frac{w\left(Z_{\ell}\right)-Z_{\ell} w^{\prime}\left(Z_{\ell}\right)}{w^{2}\left(Z_{\ell}\right)}\right) \leq C a_{m}^{q} .
$$

Combining Equations (28)-(30), we obtain

$$
\mathbb{V}\left(\frac{1}{\hat{\theta}}\right) \leq \frac{C}{n}\left(1+\sum_{m=1}^{n} a_{m}^{q}\right) \leq \frac{C}{n} .
$$

3. Using Lemma (3.1), Equation (27) and Equation (31), then

$$
\begin{aligned}
& \mathbb{E}\left(\left(\hat{\alpha}_{j, k}-\alpha_{j, k}\right)^{2}\right) \\
& \leq C\left(\mathbb{E}\left(\frac{\theta}{n} \sum_{i=1}^{n} T\left(\phi_{j, k}\right)\left(Z_{i}\right)-\alpha_{j, k}\right)^{2}+\mathbb{E}\left(\frac{1}{\hat{\theta}}-\frac{1}{\theta}\right)^{2}\right) \\
& =C\left(\mathbb{V}\left(\frac{\theta}{n} \sum_{i=1}^{n} T\left(\phi_{j, k}\right)\left(Z_{i}\right)\right)+\mathbb{V}\left(\frac{1}{\hat{\theta}}\right)\right) \leq \frac{C}{n} 2^{j(2+q)} .
\end{aligned}
$$

The proof of Proposition 3.2 is complete.

Proof of Theorem 3.1. For any integer $\ell \geq \tau$, any $f_{X} \in \mathbb{L}^{2}(0,1)$ can be expanded on $\mathcal{B}$ as 


$$
f_{X}(x)=\sum_{k=0}^{2^{j} 0-1} \alpha_{j_{0}, k} \phi_{j_{0}, k}(x)+\sum_{j=j_{0}}^{\infty} \sum_{k=0}^{2^{j}-1} \beta_{j, k} \psi_{j, k}(x),
$$

where

$$
\alpha_{j_{0}, k}=\int_{0}^{1} f_{X}(x) \phi_{j_{0}, k}(x) \mathrm{d} x, \beta_{j, k}=\int_{0}^{1} f_{X}(x) \psi_{j, k}(x) \mathrm{d} x .
$$

We obtain

$$
\mathbb{E}\left(\int_{0}^{1}\left(\hat{f}(x)-f_{X}(x)\right)^{2} \mathrm{~d} x\right)=A+B,
$$

where

$$
A=\sum_{k=0}^{2^{j_{0}}-1}\left(\left(\hat{\alpha}_{j_{0}, k}-\alpha_{j_{0}, k}\right)^{2}\right), B=\sum_{j=j_{0}}^{\infty} \sum_{k=0}^{2^{j}-1} \beta_{j, k}^{2} .
$$

Using Proposition 3.2 and inequalitity Equation (11)

$$
A \leq \frac{C}{n} 2^{j_{0}} 2^{j_{0}(2+q)} \leq C n^{-2 s /(2 s+q+3)} .
$$

and since $p \geq 2$, we have $B_{p, r}^{s}(H) \subseteq B_{2, \infty}^{s}(H)$. Hence

$$
B \leq C 2^{-2 j_{0} s} \leq C n^{-2 s /(2 s+q+3)} .
$$

Hence we have

$$
\mathbb{E}\left(\int_{0}^{1}\left(\hat{f}(x)-f_{X}(x)\right)^{2} \mathrm{~d} x\right) \leq C n^{-2 s /(2 s+q+3)} .
$$

This ends the proof of Theorem 3.1.

\section{REFERENCES}

[1] M. Carrasco and X. Chen, "Mixing and Moment Properties of Various GARCH and Stochastic Volatility Models,” Econometric Theory, Vol. 18, No. 1, 2002, pp. 17-39. doi:10.1017/S0266466602181023

[2] S. T. Buckland, D. R. Anderson, K. P. Burnham and J. L. Lake, "Distance Sampling: Estimating Abundance of Biological Populations,” Chapman and Hall, London, 1993.

[3] D. Cox, "Some Sampling Problems in Technology," In: N. L. Johnson and H. Smith Jr., Eds., New Developments in Survey Sampling, Wiley, New York, 1969, pp. 506-527.

[4] J. Heckman, "Selection Bias and Self-Selection," The New Palgrave: A Dictionary of Economics, MacMillan
Press, Stockton, 1985, pp. 287-296.

[5] R. Royall, "Statistical Evidence,” A Likelihood Paradigm, Chapman and Hall, London, 1997.

[6] R. Royall, "On the Probability of Observing Misleading Statistical Evidence,” Journal of the American Statistical Association, Vol. 95, No. 451, 2000, pp. 760-780. doi:10.1080/01621459.2000.10474264

[7] M. Emadi, J. Ahmadi and N. R. Arghami, "Comparing of Record Data and Random Observation Based on Statistical Evidence,” Statistical Papers, Vol. 48, No. 1, 2007, pp. 1-21. doi:10.1007/s00362-006-0313-z

[8] C. Chesneau and H. Doosti, "Wavelet Linear Density Estimation for a GARCH Model under Various Dependence Structures," Journal of the Iranian Statistical Society, Vol. 11, No. 1, 2012, pp. 1-21.

[9] P. Doukhan, “Mixing Properties and Examples,” Lecture Notes in Statistics 85, Springer Verlag, New York, 1994.

[10] D. Modha and E. Masry, "Minimum Complexity Regression Estimation with Weakly Dependent Observations," IEEE Transactions on Information Theory, Vol. 42, No. 6, 1996, pp. 2133-2145. doi:10.1109/18.556602

[11] A. Cohen, I. Daubechies, B. Jawerth and P. Vial, "Wavelets on the Interval and Fast Wavelet Transforms," Applied and Computational Harmonic Analysis, Vol. 24, No. 1, 1993, pp. 54-81. doi:10.1006/acha.1993.1005

[12] Y. Meyer, "Wavelets and Operators,” Cambridge University Press, Cambridge, 1992.

[13] M. Abbaszadeh, C. Chesneau and H. Doosti, "Nonparametric Estimation of Density under Bias and Multiplicative Censoring via Wavelet Methods," Statistics and Probability Letters, Vol. 82, No. 5, 2012, pp. 932-941. doi:10.1016/j.spl.2012.01.016

[14] W. Härdle, G. Kerkyacharian, D. Picard and A. Tsybakov, "Wavelet, Approximation and Statistical Applications," Lectures Notes in Statistics, Springer Verlag, New York, 1998, Vol. 129.

[15] M. Emadi and N. R. Arghami, "Some Measure of Support for Statistical Hypotheses," Journal of Statical Theory and Applications, Vol. 2, No. 2, 2003, pp. 165-176.

[16] Y. Davydov, "The Invariance Principle for Stationary Processes,” Theory of Probability \& Its Applications, Vol. 15, No. 3, 1970, pp. 498-509. doi:10.1137/1115050 